\title{
Ist bei Hydrophthalmus die Iridektomie oder die Sklerotomie als Normaloperation anzusehen?
}

Von

Dr. med. Stölting

in Hannover.

Im LXIII. Bande des Graefeschen Archivs hat Seefelder eine sehr sorgfältige Arbeit publiziert, welche den Titel trägt: Klinische und anatomische Untersuchungen zur Pathologie und Therapie des Hydrophthalmus. Es liegen der Arbeit die Erfahrungen der Leipziger Universitätsaugenklinik zugrunde, aber auch die übrigen Arbeiten auf diesem Gebiete werden darin gewürdigt. Ich möchte mich heute nur zu dem therapeutischen Teil der Arbeit äussern und eine etwas abweichende Anschauung zum Ausdruck bringen. Seefelder plädiert bei aller Anerkennung der Sklerotomieerfolge doch für die Iridektomie. Dieser Eingriff soll nach ithm die Normaloperation gegen Hydrophthalmie sein. Der Sklerotomie und Punktion der Vorderkammer, welch letztere er etwa auf die gleiche Stufe mit der Sklerotomie stellt, redet er nicht das Wort.

Könnte nicht die Seefeldersche Arbeit den Anschein erwecken, als ob die Sklerotomie in neuerer Zeit wieder verlassen sei, so wäre diese Veröffentlichung unterblieben.

Ich sollte meinen, dass in dieser Frage ganz allein die Resultate schliesslich das entscheidende; Wort zu sprechen hätten, und deshalb will ich in folgendem die meinigen mitteilen. Einige sind zwar schon früher von mir bekannt gegeben ${ }^{1}$, da aber gerade bei dieser Krank-

1) Stölting, Die Heilung der Hydrophthalmie in zwei Fällen. v. Graefe's. Arch. f. Ophth. Bd. XXXVI. 1890. - Stölting, Die Heilung der Hydrophthalmia congenita. Transact. of the 7 th internation. Congress Edinburgh 1894. 
heit auch die Dauer der Besserungen zu beobachten von Interesse ist, so führe ich die alten Fälle hier wieder mit auf.

Fall 1. Max B. Der jetzt 18jährige junge Mann kam 1888 als sieben Monate altes Kind in meine Behandlung. Damals wurde auf beiden Augen je eine Sklerotomie gemacht. Nach 14 Tagen hatte sich der Druck normalisiert. Am rechten Auge war ein halblinsengrosser Irisprolaps subconjunctival durch die nasale Ausstichöffnung entstanden. Derselbe besteht heute noch in gleicher Form. Beide Augen sind auch jetzt noch leicht vergrössert. Rechts ist die Sehschärfe bei emmetropiseher Refraktion fast $\left.{ }^{6}\right|_{4}$, links mit $+1,0$ Dioptr. $\circlearrowright+0,75$ Dioptr. Cyl. fast 6/8. Der Patient konnte bis jetzt die Schule, durch die Augen nicht behindert, besuchen. Die Optici sind gut gefärbt tund haben keine Excavation.

Fall 2. Alfred K. kam als 2jähriges Kind 1889 in meine Behandlung. Die Krankheit war weiter vorgesehritten als im Fall 1. Nach 2 Sklerotomien auf jedem Auge kam auch hier der Prozess zum Stillstand, jedoch behielten die Hornhäute einen Durchmesser von $14 \mathrm{~mm}$, auch fanden sich Trübungen, die wir heute als Rupturen der Descemetschen Membran ansprechen würden. Excavation war später nicht nachzuweisen. Anno 1895 nach $6^{1} / 2$ Jahren fand die letzte Untersuchung statt. Rechts sah der Knabe mit -12 Dioptr. ${ }^{6} / 36$ der Norm links Handbewegungen. Der Grund für die Abnahme der Sehschärfe war Amotio retinae und beginnende Katarakt am linken Auge. Leider habe ich trotz vieler Mühe keine weiteren Nachrichten von dem Patienten erhalten können.

Fall 3. Karl F. Das damals 2 jährige Kind stellte sich mir 1892 zuerst vor. Die Hydrophthalmie war weit vorgeschritten, die Corneae stark vergrössert. 2 Sklerotomien auf jedem Auge genügten die Drucksteigerung aufzuheben. Nach $11 \mathrm{Jahren}$ war der Visus rechts mit $+3,0$ Dioptr. $=3 / 60$, links mit $-3,0$ Diopt. $={ }^{6} / 60$.

Am 16. VIII. 1906 wurde mir dieser Patient, nunmehr 16 Jahre alt, aus der hiesigen Blindenanstalt zur Untersuchung vorgeführt. Er kam in Begleitung den weiten Weg gegangen, betrat aber das Sprechzimmer ohne Führung. Die beiden Augen machten einen erheblich vergrösserten Eindruck. Die Hornhautdurchmesser betrugen im horizontalen Meridian beiderseits $14 \mathrm{~mm}$.

Sehr eigentümlich war auch hier wieder die Wirkung der Sklerotomie sichtbar, und zwar wie das auch früher schon beschrieben wurde, in dem Sinne, dass die Sklerosierung des Randes nach der Operation gerade da, wo die Sklerotomie stattgefunden hatte, am weitesten vorgeschritten war. Es erschien an dieser Stelle der Rand der Hornhaut völlig abgeschrägt, er hatte seinen kreisförmigen Kontur eingebüsst. Diese Abschrägung war jedoch nur nach oben, also bei der zuerst gemachten Sklerotomie, sichtbar, nach unten konnte das nicht konstatiert werden. Rupturen der Membr. Descemetii fanden sich nicht. Die Pupillen reagierten gut, wenn auch etwas langsam auf Licht. 
Im Augenhintergrunde fiel an beiden Sehnerven die weisse Farbe ihrer äusseren Drittel auf, links war auch für diesen Teil eine schwache Excavation sichtbar, rechts nicht. Die nasalen Drittel lagen völlig im Niveau der Retina. Weitere Anomalien waren nicht zu erkennen, namentlich konnte eine Amotio retinae, wofür das Gesichtsfeld des rechten Auges sowie die etwas herabgesetzte Tension sprach, nicht gefunden werden, dagegen fiel auch der seit 3 Jahren sich immer gleich bleibende Visus dieses Auges von $3 / 60$ (mit $+2,0$ Dioptr.) ins Gewicht. Am linken Auge wurde mit $-4,0$ Dioptr. $S=4 / 50$ gefunden. Die Fixation geschah beiderseits leicht excentrisch, bei Einstellung der Augen ein wenig nach innen. Die Gesichtsfelder waren beiderseits eingeschränkt, rechts sehr wesentlich konzentrisch, links dagegen ìm Sinne der gewöhnlichen glaukomatösen Restriktion. Die Farben wurden sämtlich und ohne Zögern mit beiden Augen erkannt. Der junge Mensch beschäftigt sich mit Stuhlflechten.

Fall 4. Gottfried $K$. Der damals 10 monatliche Patient wurde mir am 9. VI. 1893 vorgestelit. Am linken Auge hatten die Eltern seit einigen Monaten die Veränderung bemerkt. Der Durchmesser der Hornhaut betrug schon $12,5 \mathrm{~mm}$ gegen $11 \mathrm{~mm}$ der rechten Seite.

Hier genügten 2 Sklerotomien, um den Druck zu normalisieren. Ich habe den Patienten regelmässig wieder gesehen, zuletzt am 22. VI. 1906, also 14 jährig; die Sehschärfe betrug am linken Ange fast ${ }^{6} / 8$ mit - 3,0 Dioptr. Das rechte Ange ist gesund geblieben. Seine Sehschärfe mit ähnlicher Korrektion $=1$. Patient ist Schüler einer höheren Schule.

Fall 5. Johann F., 11 Monate alt, aus Walsrode, einziges Kind seiner gesunden Eltern, wurde mir am 18. VIII. 1893 mit rechtsseitiger Buphthalmie gebracht. Es war von den Eltern die beginnende Vergrösserung des Auges bemerkt, als das Kind $1 / 4 \mathrm{Jahr}$ alt war. Der Prozess hatte danach bei der ersten Vorstellung mindestens 8 Monate gedauert, war allerdings nicht gänzlich unbeeinflusst geblieben, da schon einmal dem kleinen Patienten von anderer Seite Eserin verordnet wurde. Bei der Aufnahme zeigte die Hornhaut des rechten Auges einen Durchmesser von 11-12 mm, mindestens $1 \mathrm{~mm}$ mehr als die des linken. Die Randzone war noch nicht gedehnt, dagegen eine hochgradige, mit der parenchymatösen vergleichbare Hornhauttrübung vorhanden, welche die Membran fast gleichmässig, speziell aber in der Mitte trübte. Ein Einblick wurde durch dieselbe verhindert.

Noch am Tage der Aufnahme machte ich die erste Sklerotomie, woranf 2 Tage später sich die Hornhaut bis auf eine zentrale kleine Stelle aufgehelit erwies. Die Druckverhältnisse waren günstig. Einschwemmung der Iris in die Sklerotomiewunde bestand nicht, jedoch war schon am 6. Tage die Hornhaut wieder stärker getrübt nach aussen, oben begann sich auch die Uvea in der Einstichnarbe zu zeigen. Der Druck stieg, und als Eserin und warme Umschläge versagten, wurde am 27. VIII. eine zweite Sklerotomie, diesmal nach unten, ausgeführt. Auch nach dieser zweiten Operation war die Aufhellung der Cornea keine ganz vollkommene, ebensowenig konnte man sich mit der Herabsetzung des intraoku- 
laren Druckes befriedigt orklären. Schon bei dem ersten Verbandwechsel war derselbe nicht so gesunken, wie man hätte erwarten können. Immerhin zögerte ich bei fleissiger Applikation von warmen, den ganzen Tag über fortgesetzten Umschlägen und Eserin mit dem erneuten Eingriff in der Hoffnung, dass endlich die Normalisierung des Druckes eintreten sollte. Dem war jedoch nicht so; es blieb mir also nichts ïbrig, als am 17. IX. zur dritten Sklerotomie zu schreiten. Hiernach war dann zum ersten Male beim Verbandswechsel die Hornhaut völlig lzlar und der Druck sehr befriedigend. Vier Tage lang hieit sich dieser günstige Zustand, da zeigte sich wieder bei' wonigstens während der Prüfung normalem Drucke die ominöse Hornhauttrübung. Das alte Spiel begain von neuem und am 3. X. musste wieder zum operativen Eingriff geschritten werden. Ich gestehe, dass schon ein festes Vertrauen dazu gehörte, um hier nicht zu verzweifeln, jedoch krönte der Erfolg die konsequent fortgesetzten Bestrebungen. Wenn auch in der ersten Zeit noch hauchige Trübungen, ja einmal sogar am 14. X. wieder eine dentlich palpabele Drucksteigerung nachweisbar war, so konnte doch die fortschreitende Besserung nicht mehr verkannt werden. Namentlich trat das darin hervor, dass das Kind völlig ungeniert sein Auge dem licht exponierte, was um so bemerkenswerter war, als der dauernde Aufenthalt im verdunkeiten Zimmer doch zu Lichtscheu genügend Veranlassung gegeben hätte. Ebenso liess das Tränen des Anges nach und ich konnte am 19.X. dem auf die Entlassung gerichteten Wunsche der Eltern nachgeben.

Warum in diesem Falle die Operation weniger Wirksamkeit entfaltete als in den andern, ist wohl kaum zu sagen. Vielleicht war der Prozess überall ein heftigerer, wogegen allerdings der Verlauf in den ersten acht Monaten spricht, vielleicht war das Verhalten des kleinen Patienten selbst nicht ganz ohne Schuld. Als einziges Kind seiner Eltern war es mit der Pflege ausserordentlich verwöhnt, namentlich hatte die Mutter das Kind bei jeder Gelegenheit herumgetragen. Es gelang in der Klinik in diesem Falle auch nur ungenügend und spät, den kleinen Patienten besser zu gewöhnen, und das Geschrei nahm oft kein Ende. Da eine sehr befriedigende Gewichtszunahme des Kindes während der ganzen Behandlungszeit festgestellt wurde, so habe ich keinen andern Grund als schlechte Gewöhnung für die Unruhe auffinden können. Leider starb der kleine Patient im Februar 1894. Das Auge war bis zum Tode völlig klar gewesen and hatte zn keinen Besorgnissen mehr Anlass gegeben. Der Bulbus war nicht erhältlich ${ }^{1}$ ).

Fall 6. Grete St. Die Patientin stellte sich mir im Juni 1893 als 21/2 jühriges Kind vor. Das linke Auge war schon von anderer Seite vorbehandelt, und zwar mit Iridektomie. Der Zustand dieses Auges war

1) Eversbusch demonstrierte auf der Nürnberger Naturforscherversamm lung im Jahre 1893 einen Fall, wo vier rasch hintereinander folgende Sklerotomien bei einseitigem Hydrophthalmus Hoilung brachten, und zwar mit so bedeutender Reduktion der Basis, dass ein Unterschied zwischen beiden Augen nicht mehr sichtbar war. 
Ist bei Hydrophthalmus die Iridektomie oder die Sklerotomie usw. 175

trostlos; nur um dem Kinde die Schmerzen zu nehmen, konnte hier noch von operativen Eingriffen die Rede sein. Auch sie konnten schliesslich den ungünstigen Endausgang, die Enucleation, nicht aufhalten. $O b$ an dem schlechten Erfolg der Iridektomie die mangelhafte Nachbehandlung schuld war - das Kind war nach Angabe der Eltern nur 3 Tage verbunden gewesen -, wird sich nicht entscheiden lassen, jedenfalls war der Prozess hier durch Iridektomie nicht zum Stillstand gekommen, und auch die Sklerotomien hatten keinen Erfolg. Aus der Statistik der Sklerotomien muss dieses Auge schon deswegen ausscheiden, weil von Anfang an kein anderes Ziel als die Beseitigung der Schmerzhaftigkeit vorgeschwebt hatte. Bei der Vorstellung befand sich jedoch auch das rechte Auge in so vorgeschrittenem Stadium der Hydrophthalmie, dass ein hinzugezogener Augenarzt, einer der angesehensten Dentschlands, erklärte, es sei nichts zu machen, jede Operation sei abzulehnen, da sie den definitiven Verfall beschleunigen würde. Auch an diesem Auge hat sich die Methode bewährt.

Nachdem infolge von zwei durch einen Zwischenraum von 11 Tàgen getrennten Sklerotomien der Druck herabgesetzt war, blieb derselbe 120 Tage gut, dann allerdings ging er wieder in die Höhe und erforderte erneute Sklerotomie.

Ich sah das Kind zuletzt am 18. VI. 1906 wieder, es war 14jährig. Links wurde eine Prothese getragen. Der Durchmesser der rechten Hornhaut betrug $15 \mathrm{~mm}$. Die Membran erschien durch die Narben zahlreicher Sklerotomien unregelmässig begrenzt. Die Mehrzahl der Narben zeigte noch in der Mitte eine dunkle Färbung, sonst fehlte die bläuliche Übergangszone zwischen Sklera und Cornea gänzlich. Central von diesen Narben war die Hornhaut völlig durchsichtig. Es bestand geringer Grad von Iridodonesis und Nystagmus. Der Optikus war excaviert und atrophisch. Mit - 5,0 Dioptr. wurden excentrisch Finger in $3 \mathrm{~m}$ erkannt. Das Gesichtsfeld war etwa auf $1 / 5$ des Normalumfangs reduziert. Der Vater, ein Lehrer, machte hinsichtlich der Leistungen des Auges die folgenden Angaben. Das Kind hat bis zum 9. Lebensjahr in der Schule nach der Fibel lesen und schreiben gelernt, von da an aber wegen mangelnder Sehschärfe damit aufgehört. Es besucht aber auch jetzt noch die Schule regelmässig und hat in allen Nummern seines Zeugnisses die Note 2. Es schreibt und liest jetzt Blindenschrift. Auf der Strasse wird das Kind geführt, im Hanse bewegt es sich frei, es erkennt Karten zum Spielen, fängt Ball, trifft auch den vom Boden aufspringenden Ball und hilft der Mutter beim Reinigen der Zimmer.

Fall 7. Am 9. VI. 1897 wurde mir das 9 monatliche Kind, Otto H., gebracht. Beide Augen waren hydrophthalmisch, die Corneae diffus getrübt und sehr gross, Durchmesser leider nicht notiert. Ich machte die Sklerotomie auf beiden Angen, die Entlassung erfolgte am 7. Tage. Am 11. schon zeigte sich in der Sklerotomienarbe des linken Auges eine geringe ,Vordrängung der Iris. Der Druck hob sich wieder und am 44. Tage nach der Operation war ihre Wiederholung notwendig. Die Entlassung erfolgte dann nach 6 Tagen. 
Im Alter von 4 Jahren wurde eine Myopie von 5 resp. 6, Dioptr. bestimmt, Excavation war nicht vorhanden, der Druck hatte sich dauernd normal gehalten, die Corneavergrösserung blieb deutlich. Mit 5 Jahren erkannte das Kind auf $4 \mathrm{~m}$ Entfernung vorgehaltene Gegenstände. Nach einjährigem Schulbesuch wurde eine Brille notwendig. Bei den sehr unsicheren Angaben des Kindes war Prüfung mit Atropin nicht zu umgehen. Dabei stellte sich heraus, dass beiderseits nur 4 Dioptr. Myopie vorhanden waren und dass rechts $6 / 15$, links ${ }^{6}{ }_{20}$ der Norm erkannt wurde, ophthalmoskopisch war keine Andeutung von Excaration vorhanden.

Zuletzt sah ich das Kind am 20. VI. 1906, 10 jährig. Die Durchmesser der Hornhäute waren links 13, rechts $12-13 \mathrm{~mm}$. Risse der Descemetschen Membran waren beiderseits noch deutlich sichtbar, ebenso die Sklerotomienarben. Visus betrug rechts mit $-4,5$ Dioptr., links mit 6,0 Dioptr., fast $6 / 18$ der Norm Excavation nicht vorhanden.

Fall 8. Am 26. IX. 1901 wurde mir das damals 4 Monate alte Kind, Friedr. L., gebracht. Die Augen seien von der Geburt an besonders gross und schön gewesen, das rechte wäre seit 8 Tagen entzündet. An diesem Auge fand sich mässige pericorneale Injektion, rauchige Trübung der um 2-3 mm vergrösserten Hornhaut. Die Limbuszone war noch nicht namhaft verdünnt, die vordere Kammer tief, die Pupille eng; links war der Durchmesser der Hornhaut ebenso vergrössert, das Auge reizlos; die Cornea klar, Einblick war hier möglich, Details aber bei der Unruhe des Kindes nicht zu erkennen. Die beiderseits ausgeführte Sklerotomie hatte nur voribergehend den gewünschten Erfolg, 22 Tage später musste sie wiederholt werden. Auch danach stieg für kurze Zeit der Druck wieder, bis er sich etwa 45 Tage nach diesem zweiten Eingriffe normalisiert hatte. Die inzwisehen regelmässig vorgenommene Kontrolle hatte bald normalen, bald erhöhten Druck nachgewiesen. Eine Excavation war in diesem Falle nicht zu stande gekommen.

Ich habe das Kind wiederholt im Laufe der Jahre untersuchen können, niemals wieder gaben die Augen Anlass zur Sorge. Zuletzt stellte sich der kleine Patient 4jährig im Januar 1906 vor. Die Durchmesser der Hornhäute betrugen rechts 12 , links $11 \mathrm{~mm}$. Rechts waren Spuren von Zerreissung der Descemetschen Membran vorhanden, links nicht. Die Narben der Sklerotomie waren noch gut erkennbar, die Optici erschienen normal gefärbt und waren nicht excaviert. Bei Proben, mit der Hakentafel vorgenommen, ergab sich ohne Korrektion links eine Sehschärfe von $6 / 15$, rechts eine solche von $6 / 60$; dabei bestand rechts eine Myopie ron 2 Dioptr, ausserdem noch ein Astigmatismus von 2 Dioptr.

Fall 9. Am 14. Ir. 1901 sah ich zum ersten Male das 10 wöchentliche Kind, Frieda G. Die Eltern hatten gleich nach der Geburt die Abnormität der Augen bemerkt. Die Corneae waren mässig vergrössert, matt, die vordere Kammer tief. Es wurden gleich auf beiden Augen nach oben und nach 11 Tagen beiderseits nach unten Sklerotomien gemacht. Das rechte Auge erwies sich jedoch den Eingriffen sehr wenig zugänglich und ich entschloss mich weitere 4 . Wochen später zu einer Iridek- 
tomie nach oben, 12 Tage danach wurde das Kind entlassen. Als es 4 Wochen später wieder vorgestellt wurde, hatte die Hornhautektasie keinesfalls zugenommen, doch war die Membran noch so trübe, dass ein Einblick sich nicht gewinnen liess, dabei aber war wenigstens im Augenblick der Prüfung der Druck normal. Ich liess die Therapie durch Miotica und warme Umschläge fortsetzen und ordinierte Jodkali in geringen Dosen. Im Mai erwies sich der Druck ebenfalls normal, die Corneae waren aber auch da noch trübe. Eine Vergrösserung der Bulbi hatte nicht stattgefunden. Die Mutter glaubte das Kind sähe. Im September war bei der Wiedervorstellung die linke Hornhaut klar, die rechte vergrössert und trübe. Mit dem Angenspiegel konnte man den Fundus links gut sehen, rechts war das nicht möglich. Im Januar 1902 war ein ähnlicher Befund vorhanden. Die linke Cornea, völlig klar, hatte einen Durchmesser von $11-12 \mathrm{~mm}$, die rechte, völlig trübe, einen solchen von $13 \mathrm{~mm}$. Der innere Colobomschenkel, in die Narbe eingewachsen, bildete einen schwärzlichen Buckel. Eine Excavation war am linken Auge vorhanden, die nasale Hälffte der Papille erschien jedoch gut gefärbt. Es wurde nun nochmals am rechten Auge sklerotomiert mit dem Erfolg, dass bei einer Vorstellung 6 Wochen später der Druck sich normalisiert batte. Die Trübung der rechten Cornea blieb aber auch da bestehen.

Im Oktober 1902 findet sich dann in der Krankengeschichte der Vermerk: Status glaucomatosus rechts, Zustand sehr.gut links. Trotz verschiedener Aufforderungen kam das Kind nicht eher als am 23. XI. 1906 wieder. Die Mutter gab an, dass das jetzt 6 jährige Kind für die Ferne schlecht, für die Nähe dagegen gut sähe, dass es sogar Nähnadeln einfädeln könne. Dass dieser Erfolg nur mit dem linken Auge erreicht wurde, war sof̣ort klar. Seine Cornea war völlig diaphan, die Pupille, wenn auch durch einzelne Synechien etwas unregelmässig, reagierte auf Licht gut. Dagegen war der rechte Bulbus ektatisch, seine Cornea trüb, doch nicht so, dass nicht das Colobom durchgeschienen hätte. Die Sklera wies zahlreiche kleine staphylomatöse Ausbuchtungen der Limbuszone nach oben und innen oben auf. Links liess sich in beiden Meridianen Myopie nachweisen. Erst im Januar 1907 fand die proponierte Aufnahme statt. Beim Versuch am rechten Auge nach unten eine Iridektomie zu machen, trat heftige Blutung aus dem Innern des Bulbus ein. Es schoss tatsächlich das Blut im Strahl aus dem mit dem Graefeschen Messer gemachten Schnitte, so dass nichts anderes als die Enucleation tubrig blieb.

Erneute Untersuchung fand am 19. III. 1907 statt. Die Mutter gab wieder an, dass die Sehschärfe für die Nähe gut sei. Von den Untersuchungsmethoden für Refraktion gelang in diesem Falle, da ich vou Atropin absehen zu müssen glaubte, nur die im umgekehrten Bilde von Schmidt-Rimpler. Damit wurden 13 Dioptr. Myopie konstatiert. Leider kann man ja nicht sicher sagen, wieviel davon auf die Accommodation zu setzen sind, doch werden wir kaum fehlgehen, wenn wir annehmen, dass in diesem Falle eine etwas höhere als die gewöhnliche Myopie resultierte. Die temporale Hälfte des Sehnerven erwies sich als stark abgeblasst und vielleicht ein wenig excaviert. Sehprüfung konnte

v. Graefe's Archiv für Ophthalmologie. LXVIr. 1. 
leider nicht gemacht werden, da das Kind zu scheu war. Jedenfalls muss der Fall unter die günstigen Resultate für das linke Auge aufgenommen werden.

Fall 10. Die erste Vorstellung des Patienten, Hermann B., fand am 14. IV. 1903 statt. Das Kind, damals $81 / 2$ Jahre alt, wurde 2 Wochen vorher in sein linkes Auge geschlagen, welches, wie der Vater angab, von Geburt an mit einer Haut überzogen gewesen war. Wegen Tränens dieses Auges brachto man den kleinen Patienten zu mir. Es ergab sich, dass links Hydrophthalmie mit erheblicher Drucksteigerung bestand. Die Cornea war so trüb, dass nicht einmal genaues über die Vorderkammer ausgesagt werden konnte. Besonders undurebsichtig waren einzelne Partien der Membran, die wie stark trübe Bänder in den hinteren Abschnitten derselben hinzogen (Rupturen der Membrana Descemetii).

Die Sklerotomie wurde am 15. VI. 03 torgenommen, am 16. VII. findet sich die Bemerkung: linkes Auge reizlos, weich, tränt wenig, nur noch lichtempfindich. Am 23. VIII. Ange völlig reizlos. Eine Wiedervorstellung fand erst am 1. III. 07 statt. Das linke Auge war völlig geschrumpft, aber jetzt war am rechten Auge eine Vergrösserung und Tensíonserhöhung nachznweisen. Der Durchmesser der Hornhant betrug $13 \mathrm{~mm}$, nur ein leichter Hauch lag auf der Membran. Ruptur der Membrana Descemetii war, nicht vorhanden. Die Papille erwies sich bei guter Färbung als excaviert und zwar randständig nach allen Seiten. Nasal anten war das Gesichtsfeld bis auf $18^{\circ}$ eingezogen und die Sehschärfe liess sich nicht über $6 / 8$ steigern ( $-1,0$ Dioptr. cyl.). Ich muss es dahingestellt sein lassen, ob vielleicht vor 4 Jahren die ersten Spuren der Hydrophthalmie auf diesem Auge übersehen worden sind. Eine solche Deutlichkeit wie jetzt hatten sie keinenfalls.

Fall 11. Am 6. II, 1906 wurde mir die 3jährige Marie L. mit beiderseitiger Hydrophthalmie zugeführt. Nach Angabe der Eltern litt das Kind seit seiner Geburt an den Augen. Eine Anzahl von Augenärzten sei schon befragt, aber von Operationsmöglichkeit habe niemand gesprochen, vielmehr sei ihnen der Rat gegeben, das Kind einer Blindenanstalt zu überweisen. Dabei aber litt das Kind augenscheinlich an Glaukomschmerzen. Die Unruhe Tag und Nacht war für die Umgebung aufreibend, das Kind bildete eine schwere Last für die Familie. Beide Augen erschienen stark vergrössert, das linke noch erheblicher als das rechte, dabei waren die Hornhäute undurchsichtig. Noch am Tage der Aufnahme wurde an jedem Auge eine Sklerotomie ausgeführt, welche für das rechte Auge dauernd zur Normalisierung des Druckes genügte. Links war allerdings der Erfolg nur vorübergehend, am 20. II, am 7. III., am 19. III. wurden hier erneut Sklerotomien gemacht, und als auch dadurch keine danernde Herabsetzung des Druckes erzielt wurde, so folgte am 6. IV. die Iridektomie. Hiernach trat völlige Beruhigung ein. Bei einer Wiedervorstellung im Mai war der Einblick in das rechte Auge ganz deutlich, der Optikus war excaviert, dennoch gut gefärbt, am linken Auge dagegen waren die mittleren Hornhautpartien durch Rupturen der Des- 
cemetii noch getrübt. Im August hatten sich die Trübungen auch hier soweit gelichtet, dass der Einblick möglich wurde. Eine Excavation war auch hier rorhanden, doch war noeh keine Atrophie eingetreten.

Der weitere Verlauf war nicht so günstig wie ich gehofft hatte. Am 14. IV. 1906 war das Kind entlassen, am 25. IV. 1907 musste ich es wieder aufnehmen, denn der Druck am linken Auge hatte erneut zagenommen. Die Cornea war diffus trübe, im Zentrum erschien eine mehrere $\mathrm{mm}$ im Durchmesser haltende Partie grau bei unregelmässigem Epithel. Ich habe wieder eine Sklerotomie gemacht und das Kind nach 12 Tagen entlassen. Also hatte auch hier die Iridektomie versagt, wo die Sklerotomie Heilung nicht hatte bewirken können.

Am rechten Auge war dagegen der Zustand sehr befriedigend, Excavation und Abblassung der Papille waren nicht zu erkennen. Weitere Details konnte ich allerdings nicht untersuchen, da das Kind zu unruhig war. Wie mir die Mutter mitteilte, bewegt sích das Kind zu Hanse völlig allein und macht seine Besuche in der Nachbarschaft ohne Begleitung.

Man wird auch in diesem Falle wohl annehmen dürfen, dass wenigstens das rechte Auge sich erhalten wird. Suchte doch das Kind schon in der Klinik kleine Gegenstände ohne Schwierigkeiten vom Boden anf. Wie es später mit dem linken wird, halte ich allerdings für fraglich. Erneut mache ich aber auf den andern Punkt aufmerksam, der hier sehr hervortrat, die Schmerzen der unglücklichen Kinder. Nach Aussage der Elteru war die kleine Patientin von der grössten Lichtscheu geplagt, die selbst nachts nicht aufhörte. Zusammengekrümmt und wachend lag sie in ihrem Betteben und bedurfte der eingehendsten Pflege, dabei war sie im höchsten Grade unleidlich und unzugänglich. Dieses Bild änderte sich schon nach der ersten Sklerotomie. In den 69 Tagen des klinischen Aufenthalts war das Kind völlig ausgewechselt. Lichtbeständig schritt es mit erhobenem Kopf dahin, und von seiner Lebhaftigkeit erzählte das Pflegepersonal mit besonderer Vorliebe.

Die hier gefundenen Resultate stellen sich also wie folgt: In Betracht kommen 16 Augen, davon haben 8 einen Visus von ${ }^{6} / 60$ und mehr. In den beiden Fällen 9 und 11 konnte nicht geprüft werden, weil die Kinder wegen ihrer Jugend noch nichts angaben, doch müssen von den drei in Frage kommenden Augen nach der Krankengeschichte zwei gewiss zu den guten Resultaten gerechnet werden. Wir hätten demnach von 16 Augen 10 mit einem guten Erfolg. Es kämen dann noch die beiden Augen von Fall 3 in Betracht mit $5 / 60$ resp. ${ }^{5} / 60$ Sehschärfe, und vielleicht das eine Auge von Fall 11 mit Sehvermögen unter $\left.{ }^{6}\right|_{60}$. Ohne Erfolg waren schliesslich das eine Auge von Fall 9 und ein Auge von Fall 10. Bei Durchsicht der Krankengeschichten wird man sehen, dass in dem einen trotz Iridektomie das Resultat ein ungünstiges war, und in dem andern sich der 
Verlauf unserer Kenntnis entzieht, da das Kind, dem es anfangs nicht schlecht ging, sich nicht wieder vorstellte, als bis es zur Schrumpfung des Auges gekommen war. Das eine Auge von Fall 6 ist in dieser Statistik nicht mitgerechnet, weil es von vornherein keine Aussicht mehr auf Erfolg bot und nur deshalb operiert wurde, weil die Beschwerden beseitigt werden sollten. Übrigens war gerade an diesem Auge eine Iridektomie von anderer Seite gemacht worden, ehe es in meine Behandlung kam.

Ähnlich sind ja auch die von $\mathrm{Haab}$ erreichten Erfolge, welcher bekanntlich im wesentlichen mit Sklerotomie und nur in ganz schwierigen Fällen mit Iridektomie $76 \%$ gute Resultate hatte. Dem gegenüber sind von den durch Seefelder berücksichtigten Augen unter 64 bis 49 so stark amblyopisch geworden, dass ihre Sehschärfe unter ${ }^{6}{ }_{60}$ sank.

Die Vorteile der Sklerotomie gegenüber der Tridektomie treten durch diese Krankengeschichten in ein besonders helles Licht. Keine einzige Komplikation stellte sich nach Sklerotomie ein, wenn man nicht eine ektatische Narbe in Fall 1 (Visus $6 / 4$ ) als eine solche ansehen will. Dagegen bewiesen auch die Krankengeschichten der jüngsten Zeit, dass Iridektomie den üblen Ruf, welchen sie früher hatte, wenn auch nicht in dem alten Umfange, verdient.

Anscheinend angeregt durch die Freiburger Verhandlungen im Sommer 1894 hat Angelucci durch seinen Schüler Gaetano Lodato die von ihm gemachten Erfahrungen mit der Iridektomie veröftent-

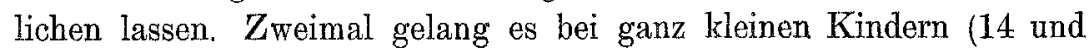
16 Tage alt), durch genannte Operation den Prozess zur Heilung zu bringen, dreimal versagte die Operation bei älteren Individuen (21 Jahre - 14 Monate - 14 Monate). Er legt das Hauptgewicht auf frühzeitiges Eingreifen, womit er ja zweifellos im Recht ist. Für die Anhänger der Iridektomie möchte ich jedoch hier kurz die Konsequenzen der erfolglosen Iridektomie, wie sie Angelucei ${ }^{1}$ ) sah, anführen.

Bei dem Fall II (21 Jahre): „Appena praticato iridectomia la cornea andò soggetta ad un processo di cheratite parenchimatosa che durò due mesi." Bei Fall IV: „Prof. A. eseguisse l'iridectomia, nell'estrarre l'iride viene fuori un po di vitreo. La visione non peggiorò, la cornea restò opaca." Bei Fall V: "Anche questo Bambino fu sottoposto all' iridectomia nell' occhio destro eseguita all' esterno con taglio sclerale, non si ebbe miglioramento. Nel praticare l'iridecto-

1) Gaetano Lodato, L'iridectomia nell' idroftalmo congenito. Archivio di ottaimologia II, p. 187. 
Ist bei Hydrophthalmus die Iridektomie oder die Sklerotomie usw. 181

mia nell" altro occhio venne fuori l'umor vitreo e si dovette riunire con punti di sutura i margini della ferita."

Mit Hilfe von Chloroform und erstarrenden Verbänden kann man heute auch relativ sicher bei dem in Rede stehenden Leiden iridektomieren. Die Heilung wird meist ungestört zu stande kommen, aber Einwachsungen der Zonula in die Wunde werden nach wie vor eintreten. Dem gegenüber ist die Sklerotomie gefahrlos. Sie genügt bei fast allen Fällen und sollte immer zuerst versucht werden. Die Verhältnisse werden sich durch Sklerotomie stets bessern, und erst wenn Tensionserhöhungen wieder und wieder eintreten, greife man zur Iridektomie. Vielleicht hat man dann noch einen Erfolg, sicher aber hat man sich durch die vorhergegangene Sklerotomie günstigere Verhältnisse geschaffen, denn die Intervalle mit herabgesetztem Druck führen schnell zur Verkleinerung des Auges und zu Verdickung der Corneoskleralzone. Die Heilung einer Iridektomiewunde wird nach voraufgegangener Sklerotomie eine viel bessere und leichtere sein als ohne sie.

Dass man hier in der Klinik Komplikationen als Folge der Sklerotomie nicht mehr fürchtet, ist übrigens zum Teil auch der Nachbehandlung mit zuzuschreiben, in welcher während der ersten Woche die Narkose eine grosse Rolle spielt. Beim ersten und eventuell beim zweiten Verbandwechsel wird das Kind, wie ich das schon in meiner ersten Arbeit beschrieben habe, chloroformiert und so lange weiter narkotisiert, bis der Verband wieder liegt. Das Kind erwacht in seinem Bett, nicht auf dem Operationstisch. Die. nachfolgende Unruhe ist eine geringe. Von den früher beschriebenen Verbandweisen ist hier nur insoweit abgewichen, als das Perlkollodium, welches von Wolffberg empfohlen wurde und sich sehr bewährt hat, für den unteren Verband und zwar ohne Heftpflaster verwandt wird. Darüber kommt der gewöhnliche Binoculus. Die Verbände liegen tagelang unberïhrt, eventuell wird nur der obere Verband gewechselt.

Ich glaube auf Grund der hiesigen Erfahrungen aussprechen zu können, dass frische Fälle von Hydrophthalmie, sofern die Degeneration noch nicht $\mathrm{zu}$ weite Fortschritte gemacht hat, im allgemeinen heilbar sind und zwar ausschliesslich durch Sklerotomie. Aber auch für veraltete Fälle genügt die Operation meistens. Keinesfalls möchte ich den negativen Standpunkt für berechtigt erklären, nach welchem jede Behandlung in den veralteten Fällen abgelehnt wird. Gerade das Gegenteil der früheren Anschauung ist richtig; die Fälle sind 
nur dann ein noli me tangere, wenn der Druck normal oder subnormal ist, aber so lange sich Drucksteigerungen finden, müssen sie operativ behandelt werden, sei es auch nur, um den unglücklichen Kindern ibre Qualen zu nehmen. Von diesem Standpunkte wird man sich auch nicht durch die theoretische Erwägung abzieben lassen dürfen, dass möglicherweise eine angeborene Obliteration der vorderen Lymphabfuhrwege der letzte Grund für die Bildung der Hydrophthalmie sein könne. Solche Fälle sind bislang klinisch nicht von den heilbaren zu unterscheiden, und die Erfahrungen lehren, dass selbst in sogenannten verzweifelten Fällen operativ noch sehr erhebliche Erfolge erzielt werden können.

Dass ich auf die statistisch gewonnenen Zahlen der Heilung nicht viel Gewicht lege, brauche ich wohl nicht hervorzuheben. Es kommt alles darauf an, in welchem Stadium die Kranken kommen. Da wird eine Klientel, welche lediglich aus Privatpatienten besteht, viel günstigere Verhältnisse darbieten, als eine Poliklinik mit ihrem oft vernachlässigt hereinkommenden Material; aber selbst wenn man diese Verhältnisse in Rechnung zieht, so wird man doch den Erfolgen, wie sie hier mitgeteilt sind, die Anerkennung nicht versagen. Natürlich bin ich weit davon entfernt, den Grund der relativ günstigen Erfolge in der Operationsmethode allein zu suchen, sondern gebe die Berechtigung der Seefelderschen Erklärung, dass man ,mit seltener Ungunst der Leipziger Verhältnisse" rechnen müsse, ohne weiteres zu, aber man kann doch aus den Resultaten nicht den Schluss ziehen, dass die Iridektomie der Sklerotomie überlegen sei.

Darüber, dass schon die Gefahren der Operation selbst bei der Iridektomie erheblich grösser sind als bei der Sklerotomie, kann doch kein $Z_{\text {weifel }}$ sein. Der Wundverschluss kann bei den verdünnten Häuten schwierig werden, die Zonula kann reissen und Glaskörper in die Wunde treten. Ferner finden Blutungen leichter statt und last not least wird beim Verbandwechsel die Wunde gelegentlich wieder eröffnet, einer der unerwünschtesten Zufälle.

Wären diese Einwürfe nicht berechtigt, wie käme es denn, dass die Iridektomie in solch schlechten Ruf geriet? Von Graefe liess eine Iridektomie nur da zu, ,wo das Übel durch Druckzunahme rasche Fortschritte macht, in der unendlichen Mehrzahl der Fälle bleibt es ein noli me tangere". Sehr deutlich erinnere ich mich noch, wie während meines Vortrags auf dem Edinburger Kongress der vor mir sitzende Ed. Meyer, bei meiner Empfehlung der Operation, wiederholt mit dem Kopfe schüttelte. Es war eben damals die Hydr- 
Ist bei Hydrophthalmus die Iridektomie oder die Sklerotomie usw. 183

ophthalmie für die unendliche Mehrzahl der Augenärzte ein noli me tangere, wie v. Graefe das ausgesprochen hatte.

Aber wie schon aus dem oben Gesagten zu schliessen ist, treten die Gefahren der Iridektomie erst dann hervor, wenn das Utbel vorgeschritten ist, leider das Stadium, in welchem wir die Patienten zumeist aufnehmen. So lange noch keine Dehnungserscheinungen an den Häuten vorhanden sind, ist die Iridektomie gefabrlos, sie ist aber auch dann erst recht unnötig, denn die Sklerotomie half in diesem Stadium bei meinen Fällen stets. Ich halte einen Menschen mit Iriscolobom nicht für verstümmelt, aber wenn man die Bildung des Coloboms vermeiden kann, soll man es doch tun. Blendungserscheinungen sind mit dem Colobom immer verbunden.

Noch möchte ich mich gegen die Ansicht wenden, dass Sklerotomie und Punktion oder Paracentese der Vorderkammer die gleiche Wirkung hätten. Führt man die Paracentese mit dem hierfür gebräuchlichen Instrument, der Paracentesennadel aus, so erhält man eine sehr schmale Wunde, die sich schon der Grösse wegen nicht mit der Sklerotomie rergleichen lässt. In der Grösse aber liegt meines Erachtens nicht einmal die Hauptsache, sondern darin, dass die Sklerotomie in der Limbuszone eine Region trifft, deren Durchschneidung, wie ich experimentell nachgewiesen habe, eine weit erheblichere Filtration ermöglicht, als eine Wunde an beliebiger Stelle näher der Hornhautmitte. Von einem Ödem der Cornea oder einzelner Teile derselben wird man allerdings nicht in allen Fällen von Hydrophthalmus sprechen können, und somit passt nicht immer die von mir gegebene Erklärung. Dafür tritt aber in den älteren Fällen die Verdünnung gerade dieser Zone ein, und auch sie wird eine länger anhaltende Filtration intraokularer Flüssigkeit nach aussen ermöglichea.

Noch sei mir gestattet, eine allgemeine Bemerkung hier anzufügen. Angesichts der günstigen Behandlungsresultate der Hydrophthalmie durch Sklerotomie kann ich mich nicht sehr dafür erwärmen, den letzten Grund für die Hydrophthalmie in einer angeborenen Obliteration des Kammerwinkels zu suchen. Es wäre doch gar nicht zu verstehen, dass dann so zahlreiche Augen auf den operativen Eingriff so überaus günstig reagierten.

Meine Erfahrungen hier stimmen auch in einem andern Punkte nicht ganz mit den Leipziger Mitteilungen überein.

Ich habe nicht konstatieren können, dass die afficierten Kinder aus besonders ärmlichen Verhältnissen stammten. Es fanden sich 
184 Stölting, Ist bei Hydrophthalmus die Iridektomie oder die Sklerotomie usw.

unter meinen Patienten ein Kind eines Oberlehrers, eines Kaufmanns, eines Volksschullehrers, eines Postboten, eines Wachtmeisters, eines Bauern, eines Fleischermeisters. Kein Kind stammte von eigentlich ganz unbemittelten Eltern. Die Beobachtung, dass die grösste Mehrzahl der Fälle Myopie aufweist, konnte auch ich bestätigen. Fs fand sich bei den von mir behandelten Augen achtmal Myopie, einmal Emmetropie und dreimal Hyperopie, von den andern Augen sind keine Angaben über Refraktion gemacht resp. waren nicht zu machen möglich. Höhere Grade von Myopie, über 10 Dioptr, wurden dabei zweimal konstatiert, der eine davon aber auch nur nach der Methode von Schmidt-Rimpler, bei der bekanntlich ohne Anwendung von Atropin die accommodative Steigerung bei Kindern kaum zu vermeiden ist. 